\title{
Complex Demodulation of Heart Rate Variability during Parabolic Flight: an Exploratory Study
}

\author{
B Verheyden, K Cockuyt, F Beckers, AE Aubert \\ Dept. of Cardiology, University Hospital Gasthuisberg, Leuven, Belgium
}

\begin{abstract}
The aim of this study was to evaluate the usefulness of complex demodulation (CDM) in assessing frequency components of heart rate variability $(H R V)$ in the setting of dynamic amplitude variations.

First, analysis of simulated data showed that the upper limit of rapid amplitude variations corresponded to a period of less than 16 s in order to allow a $95 \%$ reliable LF and HF estimation. Second, in terms of estimating the mean LF-to-HF ratio in healthy subjects, CDM was equivalent to FFT power spectrum analysis ( $r=.97$; $p<0.001)$. Finally, CDM was applied during parabolic flight to determine time-dependent responses of respiratory sinus arrhythmia (RSA) as a function of gravity. Postural differences in both heart rate and RSA were augmented during hypergravity conditions and abolished at $0 \mathrm{Gz}$ of parabolic flight.

These results show that CDM can be used to provide a continuous reliable assessment of dynamic autonomic cardiovascular control.
\end{abstract}

\section{Introduction}

The last decades, much progress has been made in understanding autonomic heart rate control through power spectral analysis (PSA) of HRV [1]. This method, however, only provides average features over the entire length of the time series and is based on the hypothesis that the system is stationary during the analysed period [2]. Recently, CDM, a time-local version of harmonic analysis, has been introduced in the field of HRV $[3 ; 4]$. CDM assumes that periodic oscillations exist within a given frequency range and quantifies the amplitude of these oscillations as a function of time [2]. Because of this characteristic, CDM enables examination of dynamic changes in the amplitude of oscillations in heart rate $[3 ; 4]$. Parabolic flight is used to create short successive periods of changing gravity in a range between $0 \mathrm{Gz}$ and $1.8 \mathrm{Gz}$ $\left(1 \mathrm{Gz}=9.81 \mathrm{~m} / \mathrm{s}^{2}\right)$ [5]. Autonomic reflex adjustments of heart rate are shown to preserve cardiovascular homeostasis during gravitational challenges in the upright position [6]. Cardiac vagal adjustments to changing gravity conditions of parabolic flight can be assessed by quantifying respiratory induced cyclic variations in heart rate (RSA) over time. However, the short duration of gravity phases $( \pm 20 \mathrm{~s})$ imposes limitations to classical HRV analysis methods [7]. We propose using CDM analysis to unravel dynamic heart rate responses during gravity transitions of parabolic flight. We first used simulated data to determine the upper limit of rapid amplitude changes that can be detected by CDM in order to evaluate the reliability of the employed CDMalgorithm. We also compared results from CDM analysis in healthy subjects to the corresponding values obtained from traditional PSA [8].

\section{Methods}

\subsection{Study protocol}

Data were obtained during the $32^{\text {nd }}$ ESA parabolic flight campaign. An AIRBUS A300 aircraft was used to perform the parabolic flight profiles (Figure 1). During 3 flights, a total of 90 parabolas were performed (30 parabolas/flight).

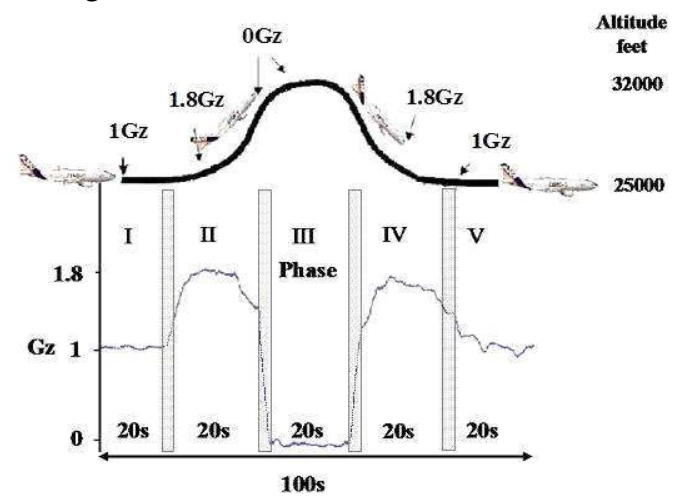

Figure 1. Schematic of the parabolic flight profiles in each flight session. Parabola phases are separated based on the instantaneous gravity level.

Three healthy male subjects (age $40 \pm 12$ yrs) were supine during the first 15 parabolas and switched to the standing position for the remaining parabolas. Breathing frequency was synchronized at $0.25 \mathrm{~Hz}$ with an auditory signal during the last 5 parabolas in each position. 
Instantaneous gravity was measured using an aircraft Gzaccelerometer. Five consecutive phases $( \pm 20 \mathrm{~s})$ were distinguished offline, based on the Gz-forces: Phase I, normogravity $(1 \mathrm{Gz})$ before each parabola; Phase II, hypergravity $( \pm 1.8 \mathrm{Gz})$ at the ascending leg of the parabola; Phase III, microgravity $( \pm 0 \mathrm{Gz})$ at the top of the parabola; Phase IV, a second period of hypergravity $( \pm 1.6 \mathrm{Gz})$ at the descending leg of the parabola; Phase $\mathrm{V}$, again normogravity $(1 \mathrm{Gz})$ after each parabola.

To examine the reliability of CDM, we compared values obtained with CDM to the corresponding values obtained from PSA in 11 healthy subjects ( 2 women) aged 19-44 yrs. Recordings were obtained during 10 minutes in standing and supine positions, preceded by 5 minutes adaptation for each position.

To determine the upper limit of rapid amplitude changes that can be detected by CDM, transfer function gain between input amplitude and demodulated amplitude of simulated data were examined [3] (Figure 2). Simulated data were generated by adding two sine waves $\left[\mathrm{A}_{1}(\mathrm{t}) \cdot \sin (2 \pi \cdot 0,09 \cdot \mathrm{t})+\mathrm{A}_{2}(\mathrm{t}) \cdot \sin (2 \pi \cdot 0,31 \cdot \mathrm{t})\right]$ with amplitudes that are modulated by two independent random signals between 0 and $0.04 \mathrm{~Hz}: \mathrm{A}_{\mathrm{i}}(\mathrm{t})=\sum_{\mathrm{k}=1}^{\mathrm{N}} 1 \cdot \cos \left(2 \cdot \lambda_{\mathrm{k}} \cdot \mathrm{t}\right)$.

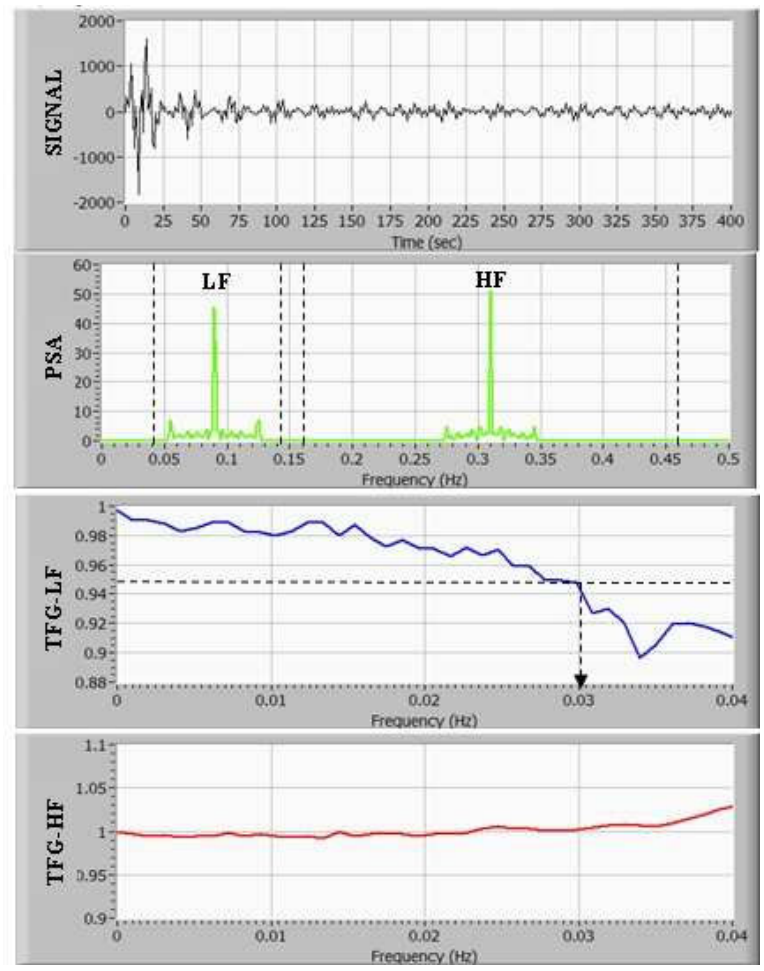

Figure 2. Upper limit of amplitude changes with CDM. From top to bottom: Input signal, power spectrum analysis (PSA), LF transfer function gain (TFG-LF), HF transfer function gain (TFG-HF).

\subsection{Data acquisition}

The ECG signal was continuously monitored and the analogue output was connected to an external A/D converter (DATAQ Instruments Inc., Akron, OH, USA). Data were sampled at $1000 \mathrm{~Hz}$ per channel and stored on a hard disc. After peak detection in the QRS-complexes, a file (tachogram) consisting of consecutive RR-intervals was created. Two linear filters could be applied to correct for data points outside a limit interval [8]. Each tachogram was resampled $(2 \mathrm{~Hz})$ using a third order cubic spline approximation [8].

\subsection{Data analysis}

Parabolic flight data were analysed using complex demodulation analysis (CDM). For the theoretical background of CDM we refer to the appendix. Briefly, $\mathrm{CDM}$ involves shifting the frequency band of interest to zero by multiplying the original signal with a complex sinusoid at the center frequency of the spectral region of interest. The resultant complex signal is then low-pass filtered and converted to a polar form to produce amplitude and phase, as a function of time. The amplitude and phase variation of CDM indicate the intensity of the signal around and the relative frequency deviation from the frequency of interest, respectively. Center frequencies of $0.09 \mathrm{~Hz}$ and $0.31 \mathrm{~Hz}$ with low-pass filter cut-off frequencies of $0.05 \mathrm{~Hz}$ and $0.15 \mathrm{~Hz}$ were selected to produce a frequency range from 0.04 to $0.14 \mathrm{~Hz}$ for the low frequency (LF) component and from 0.16 to $0.46 \mathrm{~Hz}$ for the high frequency (HF) component. Low-pass filtering was performed with a 61-term FIR-filter with Hamming window. The ratio between LF and HF (LF/HF) was calculated continuously using the instantaneous amplitudes of CDM.

To evaluate the reliability of CDM, the LF-to-HF ratio calculated with CDM was compared with corresponding values obtained with traditional power spectral analysis (PSA) [8] in 11 healthy subjects. After fulfilling stationarity criteria, the DC component was removed from each data series and the average spectrum was computed by FFT using a sliding Hanning window of 256 data points and 50\% overlap [8]. Powers in the LF and HF component were computed by integrating between appropriate frequency limits. Consequently, the ratio of LF and HF powers was calculated. All analysis software has been developed in house using Labview 7.1 (National Instruments, Austin, TX, USA) for windows.

\subsection{Statistical analysis}

The quantitative relationship between values of CDM and PSA was evaluated by linear regression analysis. The Pearson's correlation coefficient was calculated. Twoway ANOVA for repeated measurements was performed 
to examine the influence of body position throughout different gravity phases [phase I-V] of parabolic flight. A paired sample t-test was performed to evaluate postural differences within each gravity phase.

\section{Results}

Figure 2 indicates that CDM provides a $95 \%$ reliable estimate of amplitudes when the frequency of amplitude fluctuations remains below $0.03 \mathrm{~Hz}$ for $\mathrm{LF}$ and below at least $0.04 \mathrm{~Hz}$ for $\mathrm{HF}$. These values correspond to a time resolution of $<16 \mathrm{~s}$ (half of periodic oscillation) if the fluctuations in amplitude are approximated to a cosine function. The scattergram of $\mathrm{LF} / \mathrm{HF}$-ratios derived from CDM and PSA is shown in Figure 3. Results of linear regression showed that the two methods correlated well ( $\mathrm{r}$ $=.97, \mathrm{p}<0.001)$. Remark the factor 2 in the slope, which is due to the analysis: CDM decomposes amplitudes whereas PSA measures powers.

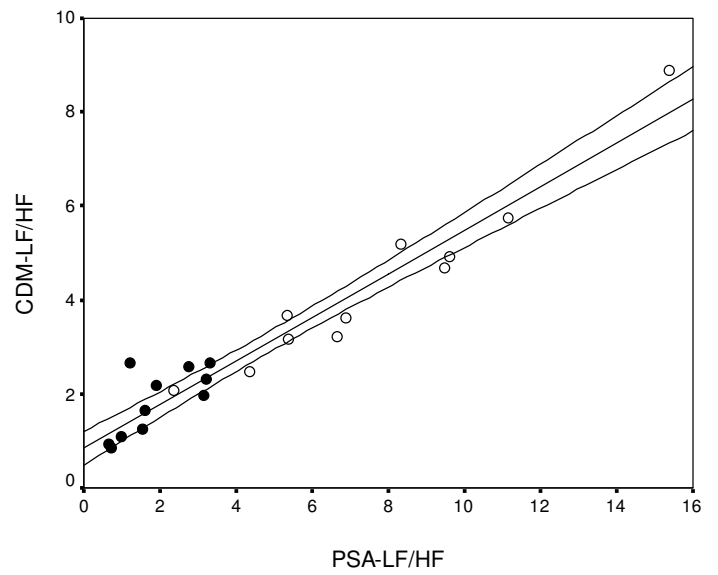

Figure 3. Linear relationship between $\mathrm{LF} / \mathrm{HF}$ derived from CDM and PSA. Open $(n=11)$ and closed $(n=33)$ circles indicate values obtained during standing and supine positions, respectively. The regression equation was $\mathrm{CDM}-\mathrm{LF} / \mathrm{HF}=0.46 \mathrm{PSA}-\mathrm{LF} / \mathrm{HF}+0.845$ with $\mathrm{R}^{2}=$ 0.93 .

According to time resolution limitations of CDM analysis (figure 2), only the last 10 s of each parabola gravity phase were selected to calculate average $\mathrm{HF}$ amplitudes of RRI fluctuations (CDM-HF) as an index of RSA. The evolution of HR and CDM-HF in standing and supine positions are depicted as function of gravity during parabolic flight in figure 4. Interestingly, postural differences that are observed at $1 \mathrm{Gz}(\mathrm{p}<0.05)$ disappear at $0 \mathrm{Gz}$, both for HR and CDM-HF. Additionally, postural differences are augmented in both hypergravity conditions $(\mathrm{p}<0.001)$. Finally, variations in $\mathrm{HR}$ and CDM-HF are most pronounced in standing position during the gravity transitions.

\section{Discussion and conclusions}

This study examined the usefulness of CDM in analysing beat to beat cardiovascular variability in a setting of dynamic autonomic heart rate control induced by changing autonomic nervous system activities. We found that $\mathrm{CDM}$ has vigorous mathematical properties that allow reliable, accurate and continuous measurement of instantaneous amplitudes of LF and HF components of HRV.
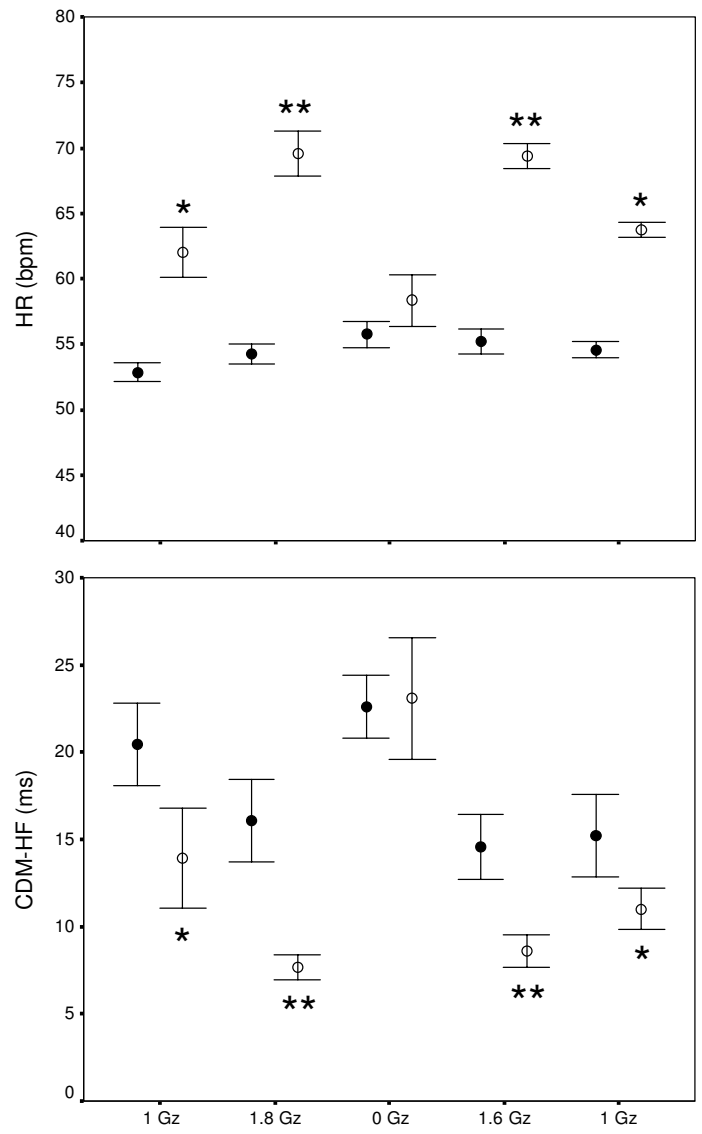

Figure 4. Evolution of HR and CDM-HF as function of gravity $(\mathrm{Gz})$ during parabolic flight. Open $(\mathrm{n}=15)$ and closed $(n=15)$ circles indicate values obtained during standing and supine positions, respectively. * $\mathrm{p}<0.05$ compared to supine; $* * \mathrm{p}<0.001$ compared to supine.

Our results from simulation data (Figure 2) indicate that, when an adequate set of filter parameters is used, CDM allows detecting amplitude changes in the LF and $\mathrm{HF}$ components of HRV with a time resolution of $<16 \mathrm{~s}$. This well exceeds the limit achieved by short-segment sequential spectrum analysis. Additionally CDM was equivalent to FFT power spectrum analysis in terms of quantifying the LF/HF ratio in healthy subjects (Figure 3 ). Hence, CDM seems to provide a flexible and reliable 
approach to assess alterations in dynamic autonomic heart rate control. This may also have important clinical applications in situations where autonomic cardiovascular control changes rapid over time, like in vasovagal syncope.

It is generally accepted that the HF component of HRV is mediated solely by vagal efferent activities and that its amplitude under frequency controlled respiration correlates with cardiac parasympathetic tone (RSA) [9]. Together with this evidence, the evolution of CDM-HF during parabolic flight supports well-known orthostatic responses of parasympathetic cardiac outflow (Figure 4). These responses include decreased parasympathetic modulation at earth gravity $(1 \mathrm{Gz})$ in standing position compared to supine. Enhanced parasympathetic control at $0 \mathrm{Gz}$ in upright position eliminated the postural differences in cardiac chronotropy. Conversely, due to greater hydrostatic pressure gradients at 1.8 and $1.6 \mathrm{Gz}$ in the upright posture, a further decrease in parasympathetic activity accentuated the postural differences in cardiac chronotropy. It should, however, be noted that due to specific time delays of sympathetic mediated alterations in cardiac chronotropy, any definitive conclusion on the CDM-LF component of HRV during parabolic flight remains difficult.

Our findings are in agreement with previous studies using other techniques of HRV analysis during parabolic flight $[6 ; 7]$. Coherent data from this study favor the validity of CDM to quantify rapid changes in cardiac parasympathetic outflow.

\section{Appendix}

According to Bloomfield [2] we can consider a time series $\quad \mathrm{X}_{\mathrm{t}}=\frac{1}{2} \mathrm{At}_{\mathrm{t}}\left[\mathrm{e}^{\{\mathrm{j} \cdot(2 \pi \cdot \lambda \cdot \mathrm{t}+\phi \mathrm{t})\}}+\mathrm{e}^{\{-\mathrm{j} \cdot(2 \pi \cdot \lambda \cdot \mathrm{t}+\phi \mathrm{t})\}}\right]+\mathrm{zt}$, where $\mathrm{A}_{\mathrm{t}}$ and $\phi_{\mathrm{t}}$ are slowly changing amplitude and phase of the component of interest, and $\mathrm{Z}_{\mathrm{t}}$ is a residual time series including other components and noise. The aim of CDM analysis is to extract approximations of $A_{t}$ and $\phi_{t}$ as a function of time. This is obtained by shifting all the frequencies in $\mathrm{X}_{\mathrm{t}}$ by $-\lambda$, which gives us $\mathrm{Yt}=\mathrm{Xt} \cdot \mathrm{e}^{-\mathrm{j} .2 \pi \cdot \lambda . \mathrm{t}}$, or $\mathrm{Yt}=\frac{1}{2} \cdot$ At.e $\mathrm{j}^{\mathrm{j} \cdot \phi \mathrm{t}}+\frac{1}{2} \cdot$ At.e $\mathrm{e}^{\{-\mathrm{j} \cdot(2 \pi \cdot 2 \lambda \cdot \mathrm{t}+\phi \mathrm{t})\}}+\mathrm{zt} \cdot \mathrm{e}^{-2 \pi \cdot \lambda \cdot \mathrm{t}}$.

The first term in the latter equation has a fluctuating frequency around zero, the second term oscillates at a frequency around $-2 \lambda$ and the third term is assumed to contain no component around zero frequency. Hence, after low-pass filtering, we obtain $\overline{\mathrm{Y}} \mathrm{t}=\frac{1}{2}$.At.e $\mathrm{j}^{\mathrm{j} \cdot \phi \mathrm{t}}$. The next step is the calculation of $A_{t}, A_{t}=2 .\left|\bar{Y}_{t}\right|$, and $\phi_{t}$, $\phi t=\tan ^{-1}\left(\frac{\operatorname{imag}\left(\frac{\bar{Y}_{t}}{\left|\bar{Y}_{t}\right|}\right)}{\operatorname{real}\left(\frac{\bar{Y}_{t}}{\left|\bar{Y}_{t}\right|}\right)}\right)$. Finally, the time dependent variation

of $\lambda \mathrm{t}$ is calculated according to $\lambda_{\mathrm{t}}=\lambda+\frac{1}{2 \pi} \cdot \frac{\mathrm{d} \phi_{\mathrm{t}}}{\mathrm{dt}}$.

\section{Acknowledgements}

This study was supported by the Belgian Federal Office of Scientific Affairs (PRODEX program: Philippe Mettens, Pierre Coquay) and B.USOC (Etienne Haumont).

\section{References}

[1] Akselrod S, Gordon D, Ubel FA, Shannon DC, Berger AC, Cohen RJ. Power spectrum analysis of heart rate fluctuation: a quantitative probe of beat-to-beat cardiovascular control. Science 1981; 213(4504):220-222.

[2] Bloomfield P. Complex demodulation. Fourier Analysis of Time Series: An Introduction. New York: Wiley, 1976: 118150.

[3] Hayano J, Taylor JA, Yamada A, Mukai S, Hori R, Asakawa $\mathrm{T}$ et al. Continuous assessment of hemodynamic control by complex demodulation of cardiovascular variability. Am J Physiol 1993; 264(4 Pt 2):H1229-H1238.

[4] Shin SJ, Tapp WN, Reisman SS, Natelson BH. Assessment of autonomic regulation of heart rate variability by the method of complex demodulation. IEEE Trans Biomed Eng $1989 ; 36(2): 274-283$.

[5] Aubert AE, Beckers F, Verheyden B, Pletzer V. What happens to the human heart in space? Parabolic flights provide some answers. ESA-Bulletin 2004; 119:30-38.

[6] Beckers F, Seps B, Ramaekers D, Verheyden B, Aubert AE. Parasympathetic heart rate modulation during parabolic flights. Eur J Appl Physiol 2003; 90(1-2):83-91.

[7] Verheyden B, Beckers F, Aubert AE. Spectral characteristics of heart rate fluctuations during parabolic flight. Eur J Appl Physiol 2005;(in press).

[8] Aubert AE, Ramaekers D, Beckers F, Breem R, Denef C, Van de WF et al. The analysis of heart rate variability in unrestrained rats. Validation of method and results. Comput Methods Programs Biomed 1999; 60(3):197-213.

[9] Hayano J, Sakakibara Y, Yamada A, Yamada M, Mukai S, Fujinami $\mathrm{T}$ et al. Accuracy of assessment of cardiac vagal tone by heart rate variability in normal subjects. Am J Cardiol 1991; 67(2):199-204.

Address for correspondence

Bart Verheyden

Laboratory of Experimental Cardiology

University Hospital Gasthuisberg O/N

Herestraat 49, 3000 Leuven, Belgium

Bart.Verheyden@med.kuleuven.be 